\title{
CORRIGENDA
}

\section{Defective DROSHA processing contributes to downregulation of MiR-15/-16 in chronic lymphocytic leukemia}

D Allegra ${ }^{1,2}$, V Bilan ${ }^{1}$, A Garding ${ }^{1,3}$, M Zucknick ${ }^{4}$, H Döhner ${ }^{1}$, S Stilgenbauer ${ }^{1}$, F Kuchenbauer ${ }^{1}$ and D Mertens ${ }^{1,2}$

Correction to: Leukemia (2014) 28, 98-107; doi:10.1038/leu. 2013.246

Since the publication of this article, the authors have identified an error concerning the author affiliation list, namely that Manuela
Zucknick was omitted from the list. The correct list of author affiliations are shown here.

The authors apologise for any inconvenience this may have caused.

${ }^{1}$ Department of Internal Medicine III, Ulm University, Ulm, Germany; ${ }^{2}$ Cooperation Unit 'Mechanisms of Leukemogenesis', German Cancer Research Center DKFZ, Heidelberg, Germany; ${ }^{3}$ Signalling to Chromatin Laboratory, Institute of Molecular Biology, Mainz, Germany and ${ }^{4}$ Department of Biostatistics, German Cancer Research Center DKFZ, Heidelberg, Germany. Correspondence: Dr D Mertens, Department of Internal Medicine III, University of Ulm, Albert-Einstein-Allee 23, Ulm 89081, Germany.

E-mail: daniel.mertens@uniklinik-ulm.de ord.mertens@dkfz.de

\section{WTAP is a novel oncogenic protein in acute myeloid leukemia}

H Bansal, Q Yihua, SP lyer, S Ganapathy, DA Proia, LO Penalva, PJ Uren, U Suresh, JS Carew, AB Karnad, S Weitman, GE Tomlinson, MK Rao, SM Kornblau and S Bansal

\begin{abstract}
Correction to: Leukemia (2014) 28, 1171-1174; doi:10.1038/ leu.2014.16
\end{abstract}

Since the publication of this article, the authors have identified an error concerning one of the author names.

D Proia should be listed as DA Proia.
The corrected list is shown above.

The Authors would like to apologise for any inconvenience this may have caused. 